\title{
The mini neutron monitor programme
}

\author{
H. Krüger ${ }^{1}$, H. Moraal, R. Nel, H.G. Krüger, M. O’Kennedy
}

Centre for Space Research, School of Physical and Chemical Sciences

North-West University, Potchefstroom, 2520, South Africa

E-mail: helena.krugerenwu.ac.za, harm.moraal@nwu.ac.za, ruan.nel42@gmail.com, hendrik.kriigeregmail.com, morgan.okennedyegmail.com

Two small neutron monitors were built in 2002 to inter-calibrate the approximately 40 stationary neutron monitors around the world, in order to study the modulation of cosmic rays derived from the resulting differential response functions. Due to electronic development during the past decade, the electronics heads were redesigned in 2011. When cheaper and more efficient counter tubes became available, the vision broadened to the concept of a mini neutron monitor, i.e. a permanent detector in its own right. Such instruments can attain count rates that approach those of standard neutron monitors if they are placed at mountain locations of > $3000 \mathrm{~m}$. Currently, three such high-altitude mini neutron monitor are operational. Another one operates at sea level, and one is used on the research vessel Polarstern to measure the instrument's latitude response, from which its rigidity response can be calculated. Measurements with these instruments are reported elsewhere in this conference.

This contribution describes the newest versions of the mini neutron monitors, i.e., the various counter tubes that are available commercially, development of the electronics, and it proposes that a more efficient second-generation network of neutron monitors can be deployed at high-altitude locations in existing buildings, using only existing infrastructure.

The 34th International Cosmic Ray Conference

30 July- 6 August, 2015

The Hague, The Netherlands

${ }^{1}$ Speaker

(c) Copyright owned by the author(s) under the terms of the Creative Commons Attribution-NonCommercial-ShareAlike Licence. 


\section{Introduction}

Neutron monitors are ground-based instruments to record the secondary particles generated by cosmic rays incident on the atmosphere. There are currently approximately 40 such neutron monitors in operation around the world, each with its own detection efficiency, due to difference in type, number of counters, and different environment.

Two small neutron monitors were built in 2002 to intercalibrate this network. The purpose was to use this intercalibration to study the modulation of cosmic rays derived from the resulting differential response functions, which are effectively the rigidity spectra of cosmic rays in the atmosphere. Such an intercalibration is needed because these spectral functions can only be derived from the normalised differences between detectors at different geomagnetic cutoff rigidities.

These detectors were called calibration neutron monitors, and their properties were described in Krüger et al., [5, 6, 7]. Recently, Amiesa et al. [1, 2] did a comprehensive numerical simulation study of this detector. The detectors have a total length of $753 \mathrm{~mm}$ and a mass of 223 $\mathrm{kg}$; see Figure 1. They are therefore mobile, and are readily transported to almost any location around the world as a unit, so that it is not necessary to dismantle them, which usually causes changes in detection efficiency. The neutron monitors at Sanae, Potchefstroom, Hermanus, Kiel and Doi Inthanon were thus calibrated.

Four developments have led to a change in vision for this programme: (a) Due to the scarcity of ${ }^{3} \mathrm{He}$ and uncontrolled spiraling of its price, the counters were getting unaffordable, (b) as a consequence, however, the regulations pertaining to the toxic ${ }^{10} \mathrm{BF}_{3}$ counters were relaxed, (c) the calibration procedure itself was difficult to manage logistically, and (d) rapid developments in electronics, in particular in interfacing technologies and data transmission, required a redesign of the electronics.
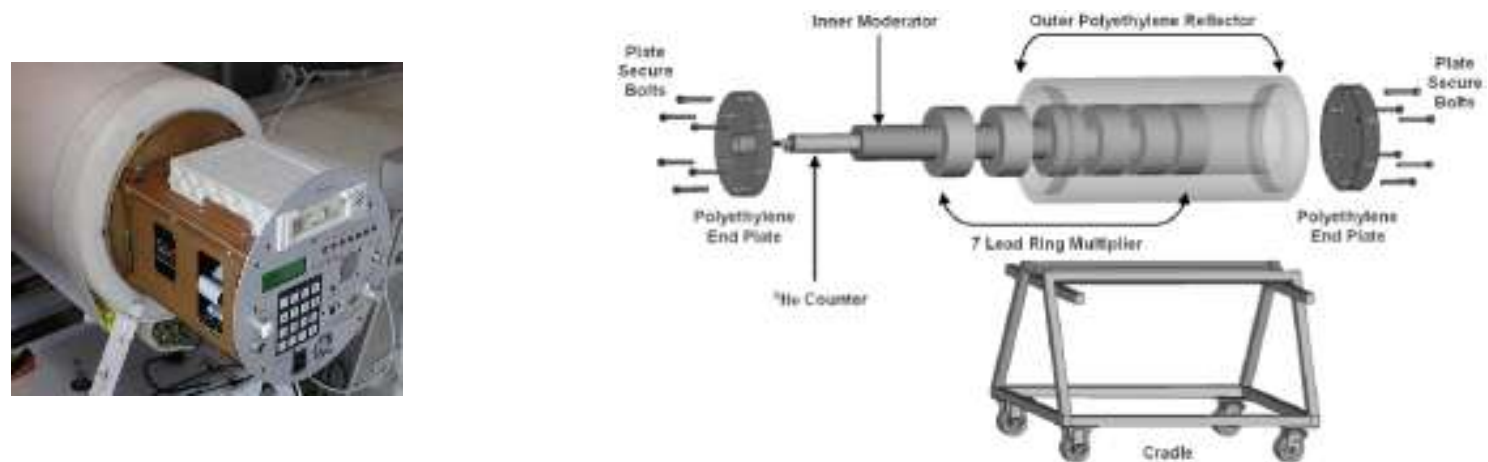

Figure 1: The original calibration neutron monitor (left) with its components (right).

This has led to a new vision, that of an affordable, permanent network of mini neutron monitors (MNM), which can approach the efficiency of the current aging network, consisting predominantly of NM64 super neutron monitors, by placing the MNMs at mountain altitudes of $>3000 \mathrm{~m}$. Currently, seven of these instruments are operational: (1) and (2) are the original ${ }^{3} \mathrm{He}$ calibrators in Potchefstroom, (3) a ${ }^{3} \mathrm{He}$ counter on the German research vessel Polarstern [4], (4) a ${ }^{10} \mathrm{BF}_{3}$ counter at the German Antarctic research base Neumayer [4], (5) and (6) two ${ }^{10} \mathrm{BF}_{3}$ counters (one with, and one without lead) at the French-Italian base Dome $\mathrm{C}$ in Antarctica at altitude 3200 m [9], and (7) on Sierra Negra mountain, Mexico, at altitude 4200 m, at the site of the High Altitude Water Cerenkov (HAWC) detector.

Progress with these instruments is reported elsewhere in the conference $[4,9]$. 
This contribution describes the newest versions of the mini neutron monitors, i.e., the various counter tubes that are available commercially, development of the electronics, cheaper pressure sensors, and it envisages a more efficient second-generation network of neutron monitors at high-altitude locations in existing buildings, using only existing infrastructure.

\section{Mini neutron monitors}

\subsection{Development of the electronics head}

Due to electronic development during the past decade, the electronics heads of the mini neutron monitors have been upgraded several times. The electronics is simple, semiautonomous; the main data storage is fully backed up by an independent high-durability flash drive, and it only needs power and network connectivity. It registers individual pulse arrival times with an accuracy of $1 \mathrm{~ms}$, from which count rates per time interval (typical resolutions minute, hour, day) are calculated. It records the normal house-keeping variables of pressure, temperature, high voltage, and GPS time and coordinates. It is inter-connectable via LAN, has a fully adaptable and versatile IP 63 system rating for protection from water and dust ingress, and has a user-friendly interface to manage and interpret data easily. Figure 2 (left) shows the current head. With a mass of $1,8 \mathrm{~kg}$ and dimensions of $10 \mathrm{~cm} \times 18 \mathrm{~cm} \times 24 \mathrm{~cm}$ it can be readily transported and exchanged.
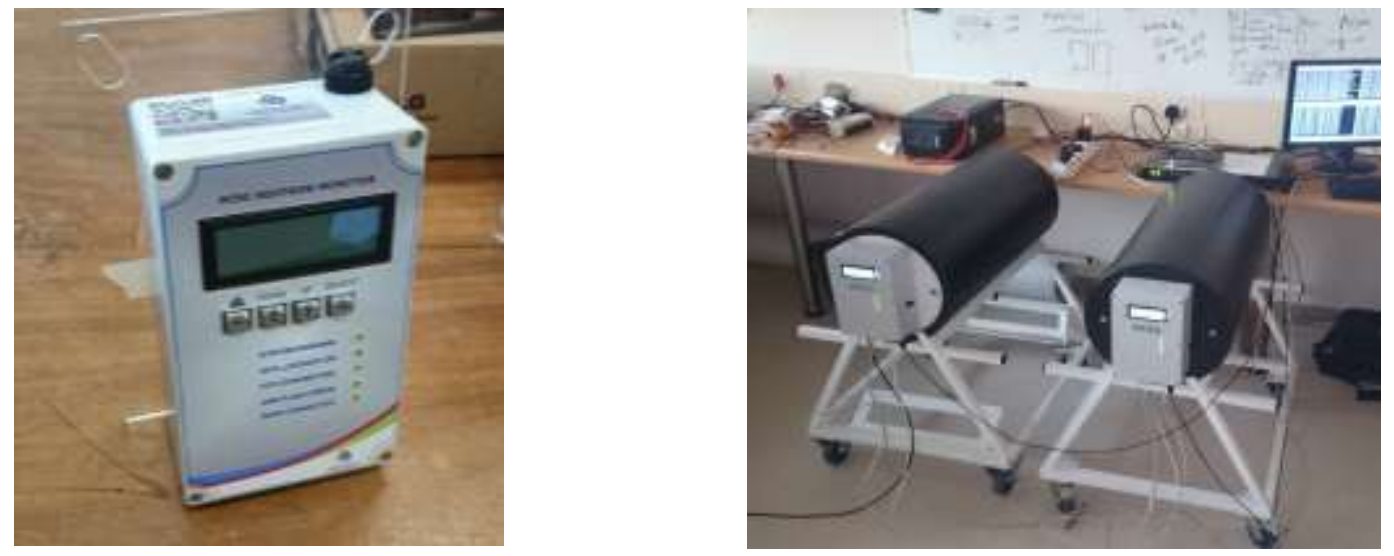

Figure 2: The front view of the upgraded electronics head (left), adapted from [3]. On the right are two mini neutron monitors on their trolleys.

The upgrades done until 2013 are described in [2].

The electronics head is also designed in such a way that it can be used on existing stationary neutron monitors. As a test case, three of the six counters of the Sanae NM64 neutron monitor, and all four counters of the lead-free neutron monitor were fitted with these heads in January 2014.

A custom-made cross-platform software package, qGraph, for data capture, transmission and analysis was developed. It is a highly interactive time-based 2D plotting and editing tool. It has a real-time graphing feature to check live NM data. It contains editing tools to correct erroneous data, combine different data sets, and various other calculation tools. At its back-end is a multiple-level sampling engine that creates graphs of various level of detail without loss of fidelity, depending on the current zoom level. Thus, it is possible to instantly plot data with time resolution of one second for up to 50 years with instantaneous zooming. 


\subsection{Counter tubes}

Table 1 shows the specifications of the various counter tubes and their count rates at sea level. The existing NM64 and IGY counters of the stationary neutron monitor network are included for comparison. The LND25382 is the ${ }^{3} \mathrm{He}$ counter used in the original calibration neutron monitor. When ${ }^{3} \mathrm{He}$ became unaffordable, we were forced to switch to the LND20366 ${ }^{10} \mathrm{BF}_{3}$ counter, losing more than a factor of 3 in count rate. In October 2011, however, the International Panel on Dangerous Goods lifted the maximum fill pressure of ${ }^{10} \mathrm{BF}_{3}$ (a toxic gas) counters from $\sim 0.3$ to $\sim 0.9$ atmospheres. This increased the count rate by almost a factor of two.

Table 1: Specifications of counters and count rates of neutron monitors.

\begin{tabular}{|c|c|c|c|c|c|c|}
\hline & \multicolumn{3}{|c|}{ Mini Neutron Monitor } & \multicolumn{2}{|c|}{ NM64 } & IGY \\
\hline Counter Type & LND25382 & LND2043 & LND20366 & LND25373 & BP28 & NW G-15-34A \\
\hline Fill Gas & ${ }^{3} \mathrm{He}$ & ${ }^{10} \mathrm{BF}_{3}$ & ${ }^{10} \mathrm{BF}_{3}$ & ${ }^{3} \mathrm{He}$ & ${ }^{10} \mathrm{BF}_{3}$ & ${ }^{10} \mathrm{BF}_{3}$ \\
\hline $\begin{array}{l}\text { Fill Pressure } \\
(\mathrm{mmHg})\end{array}$ & 3040 & 700 & 196 & 3040 & 200 & 450 \\
\hline $\begin{array}{c}\text { Operating } \\
\text { Voltage }(\mathrm{V})\end{array}$ & $1200-1450$ & $2120-2370$ & $900-1150$ & $1200-1450$ & 2800 & 1950 \\
\hline Length (mm) & 700 & 700 & 700 & 1992 & 1910 & 870 \\
\hline Diameter (mm) & 50.8 & 88.9 & 50.8 & 50.8 & 148 & 38 \\
\hline Price $(\$ \mathbf{U S})^{+}$ & $\approx 74000$ & $\approx 6000$ & $\approx 4000$ & N/A & N/A & N/A \\
\hline Counts/minute* & 84 & 63 & 25 & 660 & 660 & 61 \\
\hline
\end{tabular}

* Approximate, at sea level and cutoff rigidity $<1 \mathrm{GV}$. ${ }^{+}$The price fluctuates with at least $10 \%$ from one order to the next.

To obtain a further increase in the count rate, the volume of the counter tube was enlarged by a factor of three by increasing its diameter from $51 \mathrm{~mm}$ to $89 \mathrm{~mm}$. This resulted in model LND2043. To accommodate the larger counter, the diameter of the lead rings had to be increased, keeping the same mass. To keep the outer dimensions the same, the mass of the outer polyethylene moderator (reflector) was then decreased by $\approx 6 \%$. The last line of Table 1 shows that this configuration gives a count rate similar to the counters in the original IGY Simpson neutron monitor, and about $10 \%$ of that of an NM64 counter.

Comparison of the Sanae neutron monitor with the lead-free monitor at the same location shows that the lead multiplication factor in a standard NM64 configuration is $\approx 12$. For an LND2043 it is only $\approx 3$ to 4 , see [9]. This can be increased with additional lead, but at the expense of the easy mobility. Therefore, we rather foresee to increase the count rate by using the altitude effect, as described in section 2.4.

\subsection{Pressure sensors}

A major cost component of the registration system is a sufficiently accurate pressure sensor.

Neutron monitor variations from solar minimum to solar maximum are in the order of $20 \%$. If this range of variation is to be covered with a resolution of 100 units, it requires count rates that are accurate to $0.2 \%$. The pressure sensitivity of the count rate is of the order of $1 \%$ per $\mathrm{mm} \mathrm{Hg}$ or $0.7 \%$ per mb. Thus, the required pressure accuracy is $\pm 0.2 \mathrm{~mm} \mathrm{Hg}$ or $\pm 0.14 \mathrm{mb}$. From almost 30 years' experience with a Paroscientific Digiquartz sensor, model 760-16B, we 
could verify that is accurate and long-term stable to within $\pm 0.1 \mathrm{mb}$. However, it costs of the order of \$ US 5000.

We are therefore currently testing cheap electrostatic pressure transducers (Freescale Semiconductor, serial number MPX4115AP, price \$ US 13 each), which are based on changes in electrostatic capacitance with changes in distance between two plates, induced by pressure variations. The accuracy and stability is much worse, but the principle will be to place many of them in parallel to derive a much better average, and to eliminate those sensors that drift too strongly over time from the average.


Figure 3. Tests with cheap electrostatic pressure sensors. Left: individual sensors; right: sevensensor averages.

Figure 3 shows a test with seven such sensors, currently conducted at the Sanae neutron monitor. The two dashed lines denote a variation of $\pm 0.2 \mathrm{mb}$, which will be sufficient for count rate accurate to $0.3 \%$. The legend in the left-hand figure shows that the individual sensors started with different offsets (in units of $\mathrm{mb}$ ), and the graphs show that there is a noticeable relative drift among them over the two-month period. We also note an inverse temperature sensitivity of $\approx 0.1 \mathrm{mb} /{ }^{\circ} \mathrm{C}$. The right-hand figure shows the average of the seven sensors and the temperaturecorrected values. This corrected trace stays well within the specified accuracy of $\pm 0.2 \mathrm{mb}$.

This experiment is ongoing with other types of sensors.

\subsection{High-altitude network}

Instead of increasing the amount of lead, the count rate of the mini neutron monitor can be increased by placing it at high altitude. Atmospheric pressure increases with altitude $h$ according to $P \approx P_{0} \exp [-0.00012 h(\mathrm{~m})]$ for a standard atmosphere. With a barometric coefficient of $\approx 1$ $\% / \mathrm{mm} \mathrm{Hg}$, and $P_{0}=760 \mathrm{~mm} \mathrm{Hg}$, the count rate follows from $N=N_{0} \exp \left[0.01\left(P_{0}-P\right)\right]$, with $P$ in $\mathrm{mm} \mathrm{Hg}$. Figure 3 shows the count rate as function of altitude, indicating that the count rate at $3000 \mathrm{~m}$ is 10 times larger than at sea level. Hence, a mini NM with an LND2043 tube at 3000 $\mathrm{m}$ altitude will give the same count rate as a single-counter NM64 at sea level.

At present the two detectors of the Finnish group at Dome $\mathrm{C}$ in Antarctica are deployed at $\approx 3200 \mathrm{~m}$, with a count rate of $\approx 900$ per minute, while the one at Sierra Negra is at $4200 \mathrm{~m}$ where the multiplication factor is $\approx 20$. 


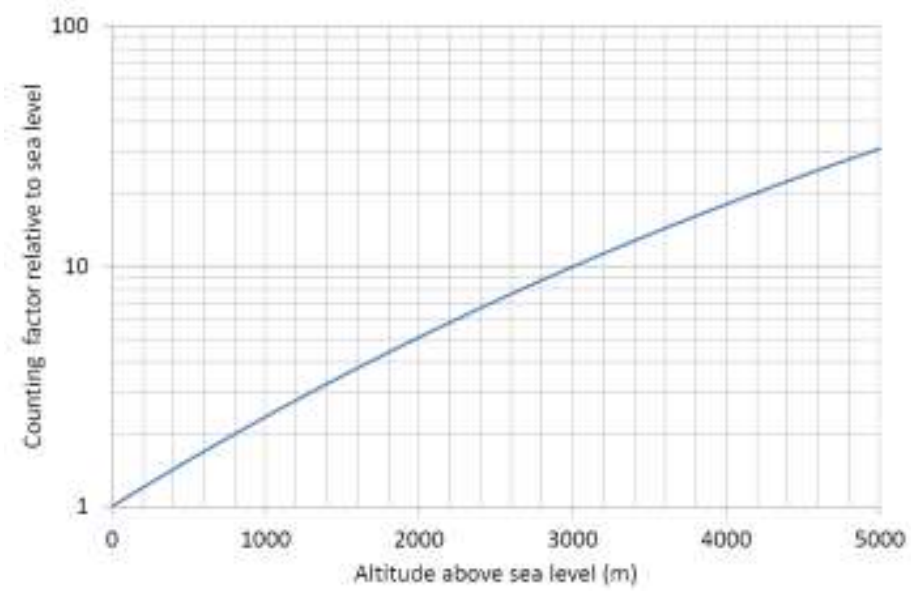

Figure 3: Count rate of a mini neutron monitor as function of altitude.

We propose to pursue this property, by thinking about a second generation of neutron monitors of this design at high altitude that can approach the efficiency of current network, which is mostly at sea level. It will evidently be much cheaper and easier to operate than the current system. It will use existing huts and other enclosures at geophysical and meteorological stations and recreational facilities. A limitation will be the number of suitable stations with power and temperature control.

\subsection{Semi-autonomous network}

To overcome this limitation, we are currently working [8] on a low-power, lowtemperature version of the mini neutron monitor which can be deployed similar to Automatic Geophysical Observatories (AGOs) in Antarctica and other unattended locations that are serviced only once per year. This requires sufficient power source and low-temperature electronics.

A typical DD-size Lithium-ion battery (twice the size of a flashlight battery) such as the SL-2790 has a capacity of $35 \mathrm{Ah} @ 3.6 \mathrm{~V}$. This is equivalent to $126 \mathrm{Wh}, 14 \mathrm{~mW}$-year, or 454 $\mathrm{kJ}$. The overall power consumption for minimal "sleep mode" of the monitor, with the minimum number of functions operating, is estimated as $42 \mathrm{Ah}$, with the three highest consumers being the Memory Control Unit $(\approx 9 \mathrm{Ah})$, the solid-state Multi-Media Card (MMC) data storage card (or SD-Card) (also $\approx 9 \mathrm{Ah}$ ), and the high-voltage supply ( $\approx 8 \mathrm{Ah}$ ). Hence, two of these batteries are sufficient to supply the system for a year.

Almost all electronic components and devices, including the Li battery, are rated for operation to temperatures as low as $-50{ }^{\circ} \mathrm{C}$. In harsh environments, such as on the high-altitude Antarctic plateau, however, temperatures can drop to $-80{ }^{\circ} \mathrm{C}$. Heat loss must therefore be prevented. The first aim is to eliminate convection, and the second to limit conduction loss. The thermal conductivity of air is $\sim 0.02 \mathrm{~W} / \mathrm{mK}$. For an insulating layer of air $10 \mathrm{~cm}$ thick, this implies that $0.02 \mathrm{~W} / \mathrm{mK} \times 10 \mathrm{~m} \times 30 \mathrm{~K}=6 \mathrm{~W}$ power is needed to sustain this temperature difference of $30 \mathrm{~K}$ over an area of $1 \mathrm{~m}^{2}$. Materials like polyurethane have similar thermal conductivity, while the best thermal insulators currently available are Vacuum Panel Insulators (VIP) with a conductivity three to four times smaller. The current electronics box has a volume of $\approx 4$ litres and surface area $\approx 0.17 \mathrm{~m}^{2}$, thus the conduction loss will be $\approx 1 \mathrm{~W}$. This will tap the 
$454 \mathrm{~kJ}$ battery in 5 days. If this temperature difference lasts 3 months per year, 18 batteries of the SL-2790 type are needed for heating.

Other limiting factors for successful operation are the mechanical robustness against low temperatures, e.g. soldering joints, brittleness of cables, and connectors.

\section{Summary}

The former calibration neutron monitor is continuously upgraded by using more efficient counter tubes, cheaper but reliable pressure sensors, and a semi-autonomous electronics head, resulting in a mini neutron monitor. The current neutron monitor work can be extended by deploying these mini neutron monitors at remote mountain stations, but the electronics heads can also be used as replacements where existing neutron monitors are threatened due to labourintensive maintenance. These affordable and portable instruments use the infrastructure that already exists at many meteorological and geophysical stations, as well as at many high-altitude mountain sites in existing buildings.

This second network with mini neutron monitors will be able to continue the concept of calibration, because the instruments are dispatched from their point of manufacture as alreadycalibrated units that are not dismantled in transport which may affect their efficiencies.

Sites can be chosen more readily than for the current network so that their asymptotic cones of acceptance can be selected more freely to make it possible to continue to observation of Ground-Level Enhancements due to solar energetic particle events.

The cost of the instrument is currently approximately \$ US 19000 . The component costs are: \$ US 6000 for the lead/polystyrene body plus trolley; \$ US 6000 for the LND 2043 counter; \$ US 5000 for the pressure transducer; and \$ US 2000 for the electronics box (including labour, but excluding development costs). If the cheap electrostatic pressure transducers prove to be sufficient, the cost will come down to approximately \$ US 13000.

\section{Acknowledgments}

We thank G.J.J. Benadé for electronic and technical improvements over a period of 5 years. This work is supported by the South African National Research Foundation Grant SNA2011110300007.

\section{References}

[1] N. Aiemsa-ad., D. Ruffolo, A. Sáiz, P.-S. Mangeard, T. Nutaro, W. Nuntiyakul, N. Kamyan, T. Khumlumlert, H. Krüger, H. Moraal, J.W. Bieber, J. Clem, P. Evenson, Measurement and simulation of neutron monitors count rate dependence on surrounding structure, Journal of Geophysical Research, Space Physics, 120, doi:10.1002/2015JA021249 (2015).

[2] N. Aiemsa-ad., D. Ruffolo, A. Sáiz, P.-S. Mangeard, T. Nutaro, W. Nuntiyakul, N. Kamyan, T. Khumlumlert, H. Krüger, H. Moraal, J.W. Bieber, J. Clem, P. Evenson, Measurement and simulation of neutron monitors count rate dependence on surrounding structure, in proceedings of the 34th ICRC (this conference), paper ID 441 (2015). 
[3] R. Fuchs, Synthesis and evaluation of a monitoring and control system for a neutron monitor, M. Eng. dissertation, North-West University (2014).

[4] B. Heber, D. Galsdorf, J. Gieseler, K. Herbst, M. Walter,A. Stoessl, H. Krüger, H. Moraal, G. Benadé, Mini neutron monitor measurements at the Neumayer III station and on the German research vessel Polarstern, in proceedings of the 34th ICRC (this conference), paper ID 1039 (2015).

[5] H. Krüger, H. Moraal, J.W. Bieber, J.M. Clem, P.A. Evenson, K.R. Pyle, M.L. Duldig and J.E. Humble, A calibration neutron monitor: energy response and instrumental temperature sensitivity, Journal of Geophysical Research, 113, A08101 doi: 10.1029/2008JA013229 (2008).

[6] H. Krüger, H. Moraal, D. Ruffolo, A. Saiz, T. Nutaro, N. Kamyan, W. Nuntiyakul, B. Heber and C. Steigies, Progress report on the intercalibration of the world's neutron monitors, in proceedings of the 32th ICRC Beijing, 11, 446-449 (2011).

[7] H. Krüger and H. Moraal, A calibration neutron monitor: statistical accuracy and environmental sensitivity, Advances in Space Research, doi:10.1016/j.asr.2010.07.008 (2010).

[8] R. Nel, Power Optimization of a Neutron Monitor System for use in a Very Low Temperature Environment. M. Eng. dissertation (in progress), North-West University (2015).

[9] I. Usoskin, S. Poluianov, H. Moraal, H. Krüger, G. Casasanta, R. Traversi, R. Udisti, A mini neutron monitor in Central Antarctica (Dome Concordia), in proceedings of the 34th ICRC (this conference), paper ID 101 (2015). 